\title{
Achievable Throughput of Multi-mode Multiuser MIMO with Imperfect CSI Constraints
}

\author{
Jun Zhang, Marios Kountouris, Jeffrey G. Andrews and Robert W. Heath Jr. \\ Wireless Networking and Communications Group \\ Department of Electrical and Computer Engineering \\ The University of Texas at Austin, Austin, TX 78712-0240 \\ Email: \{jzhang2, mkountouris, jandrews, rheath\}@ece.utexas.edu
}

\begin{abstract}
For the multiple-input multiple-output (MIMO) broadcast channel with imperfect channel state information (CSI), neither the capacity nor the optimal transmission technique have been fully discovered. In this paper, we derive achievable ergodic rates for a MIMO fading broadcast channel when CSI is delayed and quantized. It is shown that we should not support too many users with spatial division multiplexing due to the residual inter-user interference caused by imperfect CSI. Based on the derived achievable rates, we propose a multimode transmission strategy to maximize the throughput, which adaptively adjusts the number of active users based on the channel statistics information.
\end{abstract}

\section{INTRODUCTION}

For the multiple-input multiple-output (MIMO) broadcast channel, channel state information at the transmitter (CSIT) is required to separate the spatial channels for different users and achieve the full spatial multiplexing gain. CSIT, however, is difficult to get and is never perfect. Neither the capacity nor the optimal transmission technique have been fully discovered. Linear precoding combined with limited feedback [1] is a practical option, which has drawn lots of interest recently [2][5]. The main finding is that the full spatial multiplexing gain can be obtained with carefully designed feedback strategy and sufficient feedback bits that grow linearly with signal-to-noise ratio (SNR) (in $\mathrm{dB}$ ) and the number of transmit antennas.

In most systems, the number of feedback bits per user is fixed. In addition, there are other CSIT imperfections, such as estimation error and feedback delay. All of these make the system throughput limited by the residual inter-user interference at high SNR [6]. A simple approach to solve this problem is to adaptively switch between the single-user (SU) and multiuser (MU) modes, as the SU mode does not suffer from the residual interference at high SNR. SU/MU mode switching algorithms for the random beamforming system were proposed in [7], [8], where each user feeds back its preferred mode and the channel quality information. Mode switching for systems with zero-forcing (ZF) precoding and limited feedback was investigated in [9], [10], where the switching is performed during the scheduling process with properly designed channel quality information feedback.

The above mentioned SU/MU mode switching algorithms are based on instantaneous CSIT, and require feedback from each user in each time slot. In [11], [12], a SU/MU mode switching algorithm was proposed for the system with delayed and quantized CSIT. The mode switching is based on the statistics of the channel information, including the average SNR, the normalized Doppler frequency, and the codebook size, which are easily available at the transmitter. But it only switches between the SU mode and the full MU mode that serves the maximum number of users that can be supported, i.e. it is a dual-mode switching strategy.

In this paper, we consider a MIMO broadcast channel with delayed and quantized CSIT, with the amount of delay and the size of the quantization codebook fixed. We derive an achievable ergodic rate for each transmission mode, denoting the number of users served by spatial division multiplexing. It is shown that the number of active users is related to the transmit array gain, spatial multiplexing gain, and the residual inter-user interference. The full MU mode normally should not be activated, as it suffers from the highest interference while provides no array gain. A multi-mode transmission strategy is proposed to adaptively select the active mode to maximize the throughput.

\section{SySTEM MODEL}

We consider a MIMO broadcast channel with $N_{t}$ antennas at the transmitter and $U$ single-antenna receivers. Each time slot, the transmitter determines the number of users to be served, denoted as the transmission mode $M, 1 \leq M \leq N_{t}$. Eigenbeamforming is applied for the $\mathrm{SU}$ mode $(M=1)$, which is optimal with perfect CSIT. ZF precoding is used for the MU mode $\left(1<M \leq N_{t}\right)$, as it is possible to derive closed-form results due to its simple structure, and it is optimal among the set of all linear precoders at asymptotically high SNR [13]. The discrete-time complex baseband received signal at the $u$ th user in mode $M$ at time $n$ is given as

$$
y_{u}[n]=\mathbf{h}_{u}^{*}[n] \sum_{u^{\prime}=1}^{M} \mathbf{f}_{u^{\prime}}[n] x_{u^{\prime}}[n]+z_{u}[n],
$$

where $\mathbf{h}_{u}[n]$ is the channel vector for the $u$-th user, and $z_{u}[n]$ is the normalized complex additive Gaussian noise, $z_{u}[n] \sim \mathcal{C N}(0,1) . x_{u}[n]$ and $\mathbf{f}_{u}[n]$ are the transmit signal and precoding vector for the $u$-th user. The transmit power constraint is $\mathbb{E}\left[\sum_{u=1}^{M}\left|x_{u}[n]\right|^{2}\right]=P$, and we assume equal power allocation among different users. As the noise is normalized, $P$ is also the average SNR. 
To assist the analysis, we assume that the channel $\mathbf{h}_{u}[n]$ is well modeled as a spatially white Gaussian channel, with entries $h_{i}[n] \sim \mathcal{C N}(0,1)$. We assume perfect CSI at the receiver and the transmitter obtains CSI through limited feedback. In addition, there is delay in the available CSIT. The models for delay and limited feedback are presented as follows.

\section{A. CSI Delay Model}

We consider a stationary ergodic Gauss-Markov block fading regular process (or auto regressive model of order 1), where the channel stays constant for a symbol duration and changes from symbol to symbol according to

$$
\mathbf{h}[n]=\rho \mathbf{h}[n-1]+\mathbf{e}[n],
$$

where $\mathbf{e}[n]$ is the channel error vector, with i.i.d. entries $e_{i}[n] \sim \mathcal{C N}\left(0, \epsilon_{e}^{2}\right)$, and it is uncorrelated with $\mathbf{h}[n-1]$ and i.i.d. in time. We assume the CSI delay is of one symbol. For the numerical analysis, the classical Clarke's isotropic scattering model will be used as an example, for which the correlation coefficient is $\rho=J_{0}\left(2 \pi f_{d} T_{s}\right)$ with Doppler spread $f_{d}$ [14], where $T_{s}$ is the symbol duration and $J_{0}(\cdot)$ is the zeroth order Bessel function of the first kind. The variance of the error vector is $\epsilon_{e}^{2}=1-\rho^{2}$. The value $f_{d} T_{s}$ is the normalized Doppler frequency. Note that this model can also be used to model the estimation or prediction error, which makes our results more general.

\section{B. Channel Quantization}

The channel direction information is fed back using a quantization codebook known at both the transmitter and receiver. The quantization is chosen from a codebook of unit norm vectors of size $L=2^{B}$. Each user uses a different codebook to avoid the same quantization vector. The codebook for user $u$ is $\mathcal{C}_{u}=\left\{\mathbf{c}_{u, 1}, \mathbf{c}_{u, 2}, \cdots, \mathbf{c}_{u, L}\right\}$. Each user quantizes its channel direction to the closest codeword, and the closeness is measured by the inner product. Therefore, the index of channel for user $u$ is

$$
I_{u}=\arg \max _{1 \leq \ell \leq L}\left|\tilde{\mathbf{h}}_{u}^{*} \mathbf{c}_{u, \ell}\right|,
$$

where $\tilde{\mathbf{h}}_{u}=\frac{\mathbf{h}_{u}}{\left\|\mathbf{h}_{u}\right\|}$ is the channel direction. Random vector quantization (RVQ) is used to facilitate the analysis, where each quantization vector is independently chosen from the isotropic distribution on the $N_{t}$-dimensional unit sphere. The codebook based on RVQ is asymptotically optimal in probability as $N_{t}, B \rightarrow \infty$, with $\frac{B}{N_{t}} \rightarrow \hat{r} \in \mathbb{R}^{+}[15]$.

\section{Multi-mode Transmission}

In this paper, we consider a homogeneous network where all the users have the same average SNR, mobility, delay and feedback bits. The transmitter will determine how many users to serve, i.e. the active mode $M^{\star}$, and then $M^{\star}$ users are selected from the total $U$ users randomly or with round-robin scheduling. The mode selection is based on the information including average SNR, normalized Doppler frequency, and the quantization codebook size. Only the selected $M^{\star}$ users need to feed back their instantaneous channel information.
Such user scheduling is suitable for the system with scheduling independent of the channel status, such as round-robin or the one based on the queue length, or the small system with user number roughly equal to the transmit antennas, such as in a cooperative communication network.

\section{Throughrut Analysis And Mode Selection}

In this section, we derive the achievable ergodic rate for each mode, based on which the active mode can be selected. Both perfect and imperfect CSIT systems are investigated.

\section{A. Perfect CSIT}

1) $S U$ Mode (Eigen-beamforming), $M=1$ : With perfect CSIT, the beamforming (BF) vector is the channel direction vector, $\mathbf{f}[n]=\tilde{\mathbf{h}}[n]$. The ergodic achievable rate is

$$
\begin{aligned}
R_{C S I T}(1) & =R_{B F}\left(\gamma, N_{t}\right) \\
& =\mathbb{E}_{\mathbf{h}}\left[\log _{2}\left(1+\gamma\left|\mathbf{h}^{*}[n] \mathbf{f}[n]\right|^{2}\right)\right] \\
& =\log _{2}(e) e^{1 / \gamma} \sum_{k=0}^{N_{t}-1} \frac{\Gamma(-k, 1 / \gamma)}{\gamma^{k}},
\end{aligned}
$$

where $\Gamma(\alpha, x)=\int_{x}^{\infty} t^{\alpha-1} e^{-t} d t$ is the complementary incomplete gamma function. The $\mathrm{BF}$ system provides a transmit array gain $N_{t}$ as $\mathbb{E}_{\mathbf{h}}\left[\gamma\left|\mathbf{h}^{*}[n] \mathbf{f}[n]\right|^{2}\right]=N_{t} \gamma$.

2) $M U$ Mode (Zero-forcing), $1<M \leq N_{t}$ : The received SINR for the $u$-th user in a linear precoding MU-MIMO system of mode $M$ is given by

$$
\operatorname{SINR}_{u}(M)=\frac{\frac{\gamma}{M}\left|\mathbf{h}_{u}^{*}[n] \mathbf{f}_{u}[n]\right|^{2}}{1+\frac{\gamma}{M} \sum_{u^{\prime} \neq u}\left|\mathbf{h}_{u}^{*}[n] \mathbf{f}_{u^{\prime}}[n]\right|^{2}} .
$$

Denote $\mathbf{H}[n]=\left[\mathbf{h}_{1}[n], \mathbf{h}_{2}[n], \cdots, \mathbf{h}_{U}[n]\right]^{*}$, and the pseudoinverse of $\mathbf{H}[n]$ as $\mathbf{F}[n]=\mathbf{H}^{\dagger}[n]=\mathbf{H}^{*}[n]\left(\mathbf{H}[n] \mathbf{H}^{*}[n]\right)^{-1}$. The precoding vector for the $u$-th user is obtained by normalizing the $u$-th column of $\mathbf{F}[n]$. Therefore, $\mathbf{h}_{u}^{*}[n] \mathbf{f}_{u^{\prime}}[n]=$ $0, \forall u \neq u^{\prime}$, i.e. there is no inter-user interference and each user gets an equivalent interference-free channel. The SINR for the $u$-th user becomes

$$
\operatorname{SINR}_{Z F, u}(M)=\frac{\gamma}{M}\left|\mathbf{h}_{u}^{*}[n] \mathbf{f}_{u}[n]\right|^{2} .
$$

Due to the isotropic nature of i.i.d. Rayleigh fading, such orthogonality constraints to precancel inter-user interference consume $M-1$ degrees of freedom at the transmitter. As a result, the effective channel gain of each parallel channel is a chi-square random variable with $2\left(N_{t}-M+1\right)$ degrees of freedom [16], i.e. $\left|\mathbf{h}_{u}^{*}[n] \mathbf{f}_{u}[n]\right|^{2} \sim \chi_{2\left(N_{t}-M+1\right)}^{2}$. Therefore, the channel for each user is equivalent to a diversity channel with order $N_{t}-M+1$ and effective SNR $\gamma_{u}=\frac{\gamma}{M}$. The achievable rate for the $u$-th user in mode $M$ is

$$
\begin{aligned}
R_{Z F, u}\left(\gamma_{u}, M\right) & =\mathbb{E}_{\mathbf{h}}\left[\log _{2}\left(1+\frac{\gamma}{M}\left|\mathbf{h}_{u}^{*}[n] \mathbf{f}_{u}[n]\right|^{2}\right)\right] \\
& =R_{B F}\left(\gamma_{u}, N_{t}-M+1\right),
\end{aligned}
$$


where $R_{B F}(\cdot, \cdot)$ is given in (4). The achievable sum rate for the ZF system of mode $M$ is

$$
\begin{aligned}
R_{C S I T}(M) & =\sum_{u=1}^{M} R_{Z F, u}\left(\gamma_{u}, M\right) \\
& =M R_{B F}\left(\gamma_{u}, N_{t}-M+1\right) .
\end{aligned}
$$

When $M=1$, this reduces to (4).

3) Mode Selection: From (8), the system in mode $M$ provides a spatial multiplexing gain of $M$ and an array gain of $N_{t}-M+1$ for each user. As $M$ increases, the achievable spatial multiplexing gain increases but the array gain decreases. Therefore, there is a tradeoff between the achievable array gain and the spatial multiplexing gain. From (8), the mode that achieves a higher throughput for the given average SNR can be determined as

$$
M^{\star}=\arg \max _{1 \leq M \leq N_{t}} R_{C S I T}(M) .
$$

\section{B. Imperfect CSIT}

In this section, we consider a system with both delay and channel quantization. As it is difficult to derive the exact achievable rate for such a system, we provide accurate closedform approximations for mode selection.

1) SU Mode (Eigen-Beamforming): With delay and channel quantization, the $\mathrm{BF}$ vector is based on the delayed version of the quantized channel direction, $\mathbf{f}^{(Q D)}=\hat{\mathbf{h}}[n-1]$.

To get a good approximation for the achievable rate for the SU mode, we first make the following approximation on the instantaneous received SNR

$$
\begin{aligned}
\operatorname{SNR}_{B F}^{(Q D)} & =P\left|\mathbf{h}^{*}[n] \hat{\mathbf{h}}[n-1]\right|^{2} \\
& =P\left|(\rho \mathbf{h}[n-1]+\mathbf{e}[n])^{*} \hat{\mathbf{h}}[n-1]\right|^{2} \\
& \approx P \rho^{2}\left|\mathbf{h}^{*}[n-1] \hat{\mathbf{h}}[n-1]\right|^{2},
\end{aligned}
$$

i.e. we remove $\mathbf{e}[n]$, which is normally insignificant compared to $\rho \mathbf{h}[n-1]$. Eq. (10) is equivalent for a limited feedback system. From [17], the achievable rate of the limited feedback $\mathrm{BF}$ system is given by

$$
\begin{aligned}
& R_{B F}^{(Q)}(P)=\log _{2}(e)\left(e^{1 / P} \sum_{k=0}^{N_{t}-1} E_{k+1}\left(\frac{1}{P}\right)\right. \\
& \left.-\int_{0}^{1}\left(1-(1-x)^{N_{t}-1}\right)^{2^{B}} \frac{N_{t}}{x} e^{1 / P x} E_{N_{t}+1}\left(\frac{1}{P x}\right) d x\right),
\end{aligned}
$$

where $E_{n}(x)=\int_{1}^{\infty} e^{-x t} x^{-n} d t$ is the $n$-th order exponential integral. So the achievable rate of the BF system with both delay and channel quantization can be approximated as

$$
R_{B F}^{(Q D)}(P) \approx R_{B F}^{(Q)}\left(\rho^{2} P\right) .
$$

2) MU Mode (Zero-Forcing): For the MU mode with imperfect CSIT, the precoding vectors are designed based on the quantized channel directions with delay, which achieve $\hat{\mathbf{h}}_{u}^{*}[n-1] \mathbf{f}_{u^{\prime}}^{(Q D)}[n]=0, \forall u \neq u^{\prime}$. The SINR for the $u$-th user in mode $M$ is given as

$$
\gamma_{Z F, u}^{(Q D)}(M)=\frac{\frac{P}{M}\left|\mathbf{h}_{u}^{*}[n] \mathbf{f}_{u}^{(Q D)}[n]\right|^{2}}{1+\frac{P}{M} \sum_{u^{\prime} \neq u}\left|\mathbf{h}_{u}^{*}[n] \mathbf{f}_{u^{\prime}}^{(Q D)}[n]\right|^{2}} .
$$

Following Theorem 3 in [11], the average residual inter-user interference for the $u$-th user is

$$
\begin{aligned}
& \mathbb{E}_{\mathbf{h}}\left[\frac{P}{M} \sum_{u^{\prime} \neq u}\left|\mathbf{h}_{u}^{*}[n] \mathbf{f}_{u^{\prime}}^{(Q D)}[n]\right|^{2}\right] \\
= & \left(1-\frac{1}{M}\right) P\left(\rho_{u}^{2} \frac{N_{t}}{N_{t}-1} 2^{-\frac{B}{N_{t}-1}}+\epsilon_{e, u}^{2}\right) .
\end{aligned}
$$

Remark 1: The residual interference depends on delay, codebook size, $N_{t}$, and $M$. It increases with delay, and decreases with the codebook size. At high SNR, it makes the system interference-limited. With other parameters fixed, residual interference increases as $M$ increases, and mode selection will take this into consideration.

To approximate the achievable ergodic rate, we first approximate the signal term as

$$
\begin{aligned}
P_{S} & =\frac{P}{M}\left|\mathbf{h}_{u}^{*}[n] \mathbf{f}_{u}^{(Q D)}[n]\right|^{2} \\
& =\frac{P}{M}\left|\left(\rho_{u} \mathbf{h}_{u}[n-1]+\mathbf{e}_{u}[n]\right)^{*} \mathbf{f}_{u}^{(Q D)}[n]\right|^{2} \\
& \stackrel{(a)}{\approx} \frac{P}{M}\left|\rho_{u} \mathbf{h}_{u}^{*}[n-1] \mathbf{f}_{u}^{(Q D)}[n]\right|^{2} \\
& \stackrel{(b)}{\approx} \frac{P}{M}\left|\rho_{u}\left\|\mathbf{h}_{u}[n-1]\right\| \hat{\mathbf{h}}_{u}^{*}[n-1] \mathbf{f}_{u}^{(Q D)}[n]\right|^{2},
\end{aligned}
$$

where step (a) removes $\mathbf{e}_{u}^{*}[n] \mathbf{f}_{u}^{(Q D)}[n]$, which is normally very small. Step (b) approximates the actual channel direction by the quantized version, which is justified for small quantization error. As $\hat{\mathbf{h}}_{u}[n-1]$ is independent from other users, similar to the system with perfect CSIT, $\left\|\mathbf{h}_{u}[n-1]\right\| \cdot \mid \hat{\mathbf{h}}_{u}^{*}[n-$ $1]\left.\mathbf{f}^{(Q D)}[n]\right|^{2} \sim \chi_{2\left(N_{t}-M+1\right)}^{2}$.

The received SINR for the $u$-th user can then be approximated as in (16), on the top of the next page. The approximation for the denominator comes from removing the terms with both $\mathbf{e}_{u}[n]$ and $\mathbf{f}_{u^{\prime}}^{(Q D)}[n]$. For the interference terms in (16), as $\mathbf{e}_{u}[n] \sim \mathcal{C N}\left(\mathbf{0}, \epsilon_{e, u}^{2} \mathbf{I}\right),\left|\mathbf{f}_{u^{\prime}}[n]\right|^{2}=1$, and they are independent, $\left|\mathbf{e}_{u}^{*}[n] \mathbf{f}_{u^{\prime}}[n]\right|^{2}$ is an exponential random variable with mean $\epsilon_{e}^{2}$. The following lemma in [12] provides the distribution of the term $\left|\mathbf{h}_{u}^{*}[n-1] \mathbf{f}_{u^{\prime}}^{(Q D)}[n]\right|^{2}$.

Lemma 1: Based on the quantization cell approximation [5], the interference term due to quantization, $\mid \mathbf{h}_{u}^{*}[n-$ 1] $\left.\mathbf{f}_{u^{\prime}}^{(Q D)}[n]\right|^{2}$, can be approximated as an exponential random variable with mean $\delta=2^{-\frac{B}{N_{t}-1}}$.

Remark 2: The residual interference terms due to both delay and quantization are exponential random variables, which means the delay and quantization error have equivalent effects but with different means.

Based on the distributions of the interference terms, we can get the following theorem on the achievable rate for the MU mode. 


$$
\begin{gathered}
\gamma_{Z F, u}^{(Q D)} \approx \frac{\frac{P}{M} \rho^{2}\left\|\mathbf{h}_{u}[n-1]\right\| \cdot\left|\hat{\mathbf{h}}_{u}^{*}[n-1] \mathbf{f}^{(Q D)}[n]\right|^{2}}{1+\frac{P}{M} \sum_{u^{\prime} \neq u} \rho^{2}\left|\mathbf{h}_{u}^{*}[n-1] \mathbf{f}_{u^{\prime}}^{(Q D)}[n]\right|^{2}+\frac{P}{M} \sum_{u^{\prime} \neq u}\left|\mathbf{e}_{u}^{*}[n] \mathbf{f}_{u^{\prime}}[n]\right|^{2}} \\
R_{Q D, u}(M) \approx \log _{2}(e) \sum_{i=0}^{N_{t}-L-1} \sum_{j=1}^{2} \sum_{k=0}^{L-1} \sum_{l=0}^{i} \frac{a_{k}^{(j)}(l+k) !}{l !(i-l) !} \alpha^{l+k-i+1} \hat{I}\left(\frac{1}{\alpha}, \frac{\alpha}{\delta_{j}}, i, l+k+1\right) \\
R_{D, u}(M) \approx \log _{2}(e) \sum_{i=0}^{N_{t}-L-1} \sum_{l=0}^{i}\left(\begin{array}{c}
L+l-1 \\
l
\end{array}\right) \frac{\alpha^{L+l-i}}{\beta^{L}(i-l) !} \cdot \hat{I}\left(\frac{1}{\alpha}, \frac{\alpha}{\beta}, i, L+l\right)
\end{gathered}
$$

Theorem 1: The achievable ergodic rate for the $u$-th user in mode $M(M>1)$ with both delay and channel quantization can be approximated by (17), where $\alpha=\frac{\rho_{u}^{2} P}{M}, \delta_{1}=\frac{\rho_{u}^{2} P \delta}{M}$, $\delta_{2}=\frac{\epsilon_{e, u}^{2} P}{M}, L=M-1, \hat{I}$ is the following integral

$$
\hat{I}(a, b, m, n)=\int_{0}^{\infty} \frac{x^{m} e^{-a x}}{(x+b)^{n}(x+1)} d x,
$$

and

$$
\begin{aligned}
& a_{i}^{(1)}=\frac{\mathcal{A}}{\delta_{1}^{i+1}}\left(\frac{\delta_{1}}{\delta_{1}-\delta_{2}}\right)^{L}\left(\frac{\delta_{2}}{\delta_{2}-\delta_{1}}\right)^{L-1-i}, \\
& a_{i}^{(2)}=\frac{\mathcal{A}}{\delta_{2}^{i+1}}\left(\frac{\delta_{2}}{\delta_{2}-\delta_{1}}\right)^{L}\left(\frac{\delta_{1}}{\delta_{1}-\delta_{2}}\right)^{L-1-i}
\end{aligned}
$$

with $\mathcal{A}=\frac{1}{(L-1) !} \frac{(2(L-1)-i) !}{i !(L-1-i) !}$.

Proof: This approximation is derived by assuming the interference terms are pair-wise independent, and also independent of the signal term. Then the CDF of the SINR can be derived, following which the achievable rate can be calculated. A closed form expression can be derived for $\hat{I}(a, b, m, n)$, which is not provided due to space limitation.

As a special case, with only delay, following (15) and (16) the received SINR for the $u$-th user is approximated as

$$
\gamma_{Z F, u}^{(D)} \approx \frac{\frac{P}{M}\left|\rho_{u} \mathbf{h}_{u}^{*}[n-1] \mathbf{f}_{u}[n]\right|^{2}}{1+\frac{P}{M} \sum_{u^{\prime} \neq u}\left|\mathbf{e}_{u}^{*}[n] \mathbf{f}_{u^{\prime}}[n]\right|^{2}},
$$

for which the achievable ergodic rate is provided as follows.

Corollary 1: The achievable ergodic rate for the $u$-th user in the delayed system of mode $M(M>1)$ can be approximated by (18), where $\alpha=\frac{\rho_{u}^{2} P}{M}, \beta=\frac{\epsilon_{e, u}^{2} P}{M}, L=M-1$, and $\hat{I}$ is the integral in (19).

From Lemma 7 the effects of delay and channel quantization are equivalent, so the result in this corollary also applies for the limited feedback system, replacing $\epsilon_{e, u}^{2}$ by $\delta=2^{-\frac{B}{N_{t}-1}}$.

\section{Mode Selection}

Based on (12) and (17), the active mode in the system with both delay and channel quantization is selected according to

$$
M^{\star}=\arg \max _{1 \leq M \leq N_{t}} R_{Q D}(M),
$$

where $R_{Q D}(M)=\sum_{u=1}^{M} R_{Q D, u}(M)$.

Remark 3: Considering (14), (15), and (17), the mode $M$ is now related to the residual interference, the transmit array gain, and the spatial multiplexing gain. The multi-mode transmission is to balance between these effects to improve the system throughput.

\section{Numerical Results: VerificAtion of ANALysis AND KEY OBSERVATIONS}

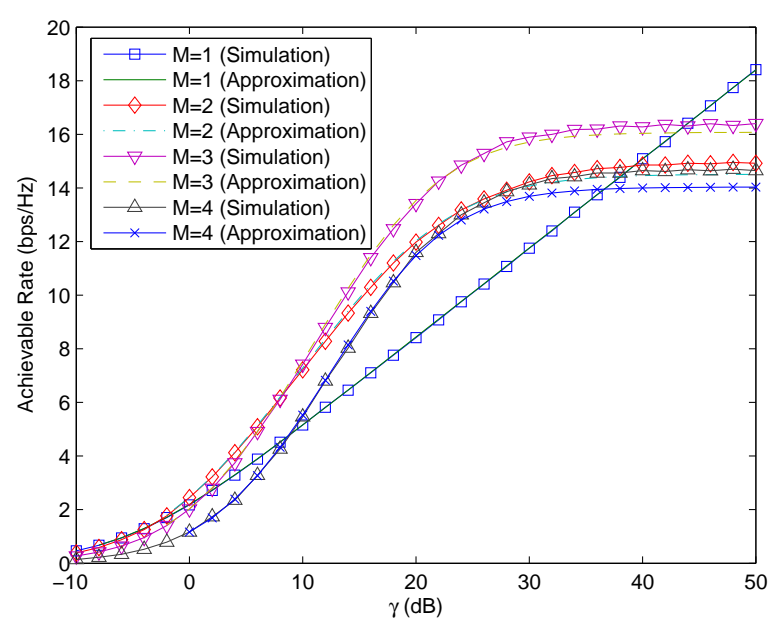

Fig. 1. Comparison of simulations and approximations for different $M$, $N_{t}=4$, mobility $v=10 \mathrm{~km} / \mathrm{hr}, T_{s}=1 \mathrm{msec}, B=18$.

We first verify the derived approximations for the achievable rates in Fig. 11 We see that the approximation is very accurate at low to medium SNRs. At high SNR, the approximation becomes a lower bound, and the accuracy decreases as $M$ increases. Interestingly, we see that the mode $M=3$ always provides a higher throughput than the full MU mode $M=4$. This is due to the fact that the full mode has the highest level of residual interference while provides no array gain. Therefore, it is desirable to do spatial division multiplexing for fewer than $N_{t}$ users, which provides addition array gain and reduces the residual interference for each user. We see that the proposed multi-mode transmission provides a significant throughput gain over the SU-MIMO system $(M=1)$. In addition, it is able to provide a throughput gain around 2 $\mathrm{bps} / \mathrm{Hz}$ over the dual-mode switching [11], [12] at medium SNR.

From (21), we can determine the active mode $M^{\star}$ for a given scenario. Accordingly, the operating regions for different 


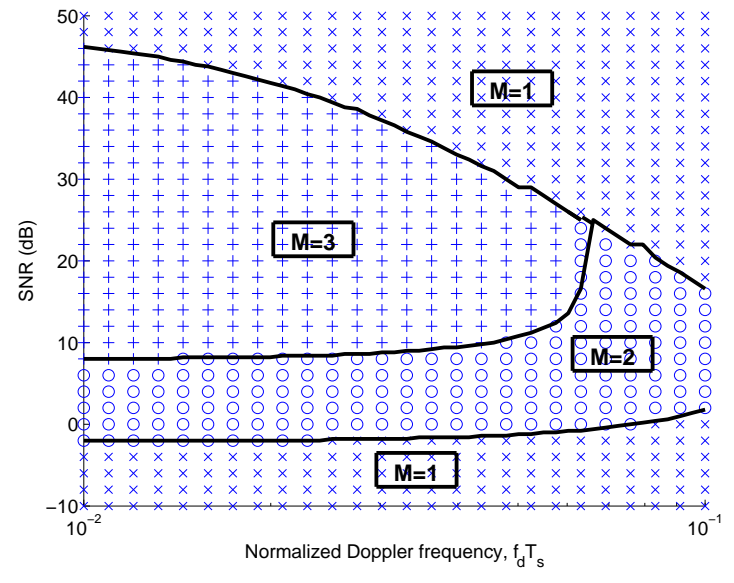

(a) Different $f_{d} T_{s}, B=18$.

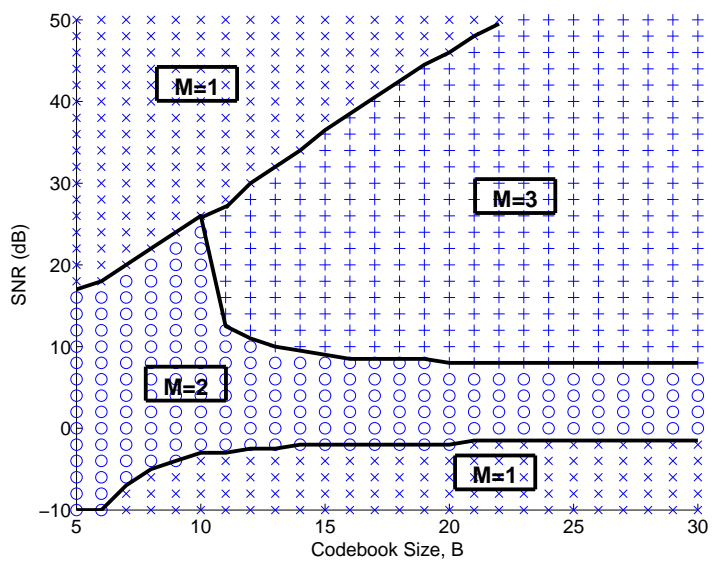

(b) Different $B, f_{c}=2 G H z, T_{s}=1 \mathrm{msec}$, and $v=10 \mathrm{~km} / \mathrm{hr}$.

Fig. 2. Operating regions for different modes with both CSI delay and channel quantization, $U=N_{t}=4$. The mode $M=i$ means that there are $i$ active users. In this plot, ' $\mathrm{X}$ ' is for $M=1$, ' $\mathrm{o}$ ' is for $M=2$, ' + ' is for $M=3$, and ' $\square$ ' is for $M=4$.

modes can be plotted for different system parameters. Fig.2(a) and Fig. 2(b) show the operating regions for different $f_{d} T_{s}$ and different $B$, respectively. There are several key observations.

(i) For the given $f_{d} T_{s}$ and $B$, the $\mathrm{SU}$ mode $(M=1)$ will be active at both low and high SNRs, due to its array gain and the robustness to imperfect CSIT, respectively.

(ii) For higher MU modes to be active, $f_{d} T_{s}$ needs to be small while $B$ needs to be large. Specifically, to get the mode $M=3$ activated, we need $f_{d} T_{s}<0.57$ with $B=18$ as in Fig. 2(a), and need $B>10$ with $v=10$ $\mathrm{km} / \mathrm{hr}, f_{c}=2 \mathrm{GHz}$, and $T_{s}=1 \mathrm{msec}$ as in Fig. 2(b).

(iii) The highest mode $M=N_{t}$ is not active with the considered parameters. We find that it is possible to be active only when $f_{d} T_{s} \lesssim 0.01$ and $B$ is large enough.

\section{CONCLUSIONS}

In this paper, we derive the achievable ergodic rates for the MIMO broadcast channel with imperfect CSIT. Due to residual inter-user interference, it is not desirable to serve too many users with spatial division multiplexing. Based on the derived rates, a multi-mode transmission strategy is proposed that adaptively adjusts the number of active users. It is based on the channel statistics information, and only the selected users need to feed back the instantaneous channel information.

\section{REFERENCES}

[1] D. J. Love, R. W. Heath Jr., V. K. N. Lau, D. Gesbert, B. D. Rao, and M. Andrews, "An overview of limited feedback in wireless communication systems," IEEE J. Select. Areas Commun., vol. 26, no. 8, pp. 1341-1365, Oct. 2008 .

[2] N. Jindal, "MIMO broadcast channels with finite rate feedback," IEEE Trans. Inform. Theory, vol. 52, no. 11, pp. 5045-5059, Nov. 2006.

[3] G. Caire, N. Jindal, M. Kobayashi, and N. Ravindran, "Multiuser MIMO downlink made practical: Achievable rates with simple channel state estimation and feedback schemes," Submitted to IEEE Trans. Information Theory, available online at http://arxiv.org/pdf/0711.2642.

[4] P. Ding, D. J. Love, and M. D. Zoltowski, "Multiple antenna broadcast channels with partial and limited feedback," IEEE Trans. Signal Processing, vol. 55, no. 7, pp. 3417-3428, Jul. 2007.
[5] T. Yoo, N. Jindal, and A. Goldsmith, "Multi-antenna downlink channels with limited feedback and user selection," IEEE J. Select. Areas Commun., vol. 25, no. 7, pp. 1478-1491, Sept. 2007.

[6] A. Lapidoth, S. Shamai, and M. Wigger, "On the capacity of fading MIMO broadcast channels with imperfect transmitter side-information," in Proceedings of the 43rd Allerton Conference on Communication, Control and Computing, Monticello, IL, Sept. 2005.

[7] C. K. Au-Yeung, S. Y. Park, and D. J. Love, "A simple dual-mode limited feedback multiuser downlink system," accepted for publication in IEEE Trans. Comm.

[8] M. Kountouris, D. Gesbert, and T. Salzer, "Distributed transmit mode selection for MISO broadcast channels with limited feedback: Switching from SDMA to TDMA," in Proc. IEEE Workshop on Signal Processing Advances in Wireless Communications (SPAWC 2008), Recife, Brazil, Jul. 2008, pp. 371-375.

[9] M. Kountouris, R. de Francisco, D. Gesbert, D. T. M. Slock, and T. Salzer, "Efficient metrics for scheduling in MIMO broadcast channels with limited feedback," in Proc. of the IEEE Int. Conf. on Acoustics, Speech, and Signal Proc., Honolulu, USA, Apr. 2007, pp. 109-112.

[10] M. Trivellato, F. Boccardi, and F. Tosato, "User selection schemes for MIMO broadcast channels with limited feedback," in Proc. IEEE Veh. Technol. Conf., Dublin, Ireland, Apr. 2007.

[11] J. Zhang, J. G. Andrews, and R. W. Heath Jr., "Single-user MIMO vs. multiuser MIMO in the broadcast channel with CSIT constraints," in Proc. of Allerton Conf. on Comm. Control and Comp., Monticello, IL, Sept. 2008.

[12] J. Zhang, R. W. Heath Jr., M. Kountouris, and J. G. Andrews, "Mode switching for MIMO broadcast channel based on delay and channel quantization," submitted to EURASIP Special Issue on Multiuser MIMO Transmission with Limited Feedback, Cooperation, and Coordination, available at http://users.ece.utexas.edu/ jzhang2/.

[13] N. Jindal, "A high SNR analysis of MIMO broadcast channels," in Proc. IEEE Int. Symp. Information Theory, Adelaide, Australia, Sept. 2005, pp. 2310-2314.

[14] R. H. Clarke, "A statistical theory of mobile radio reception," Bell System Tech. J., vol. 47, pp. 957-1000, 1968.

[15] W. Dai, Y. Liu, and B. Rider, "Quantization bounds on Grassmann manifolds and applications in MIMO systems," IEEE Trans. Inform. Theory, vol. 54, no. 3, pp. 1108-1123, Mar. 2008.

[16] J. Lee and N. Jindal, "High SNR analysis for MIMO broadcast channels: Dirty paper coding vs. linear precoding," IEEE Trans. Inform. Theory, vol. 53, no. 12, pp. 4787-4792, Dec. 2007.

[17] C. K. Au-Yeung and D. J. Love, "On the performance of random vector quantization limited feedback beamforming in a MISO system," IEEE Trans. Wireless Commun., vol. 6, no. 2, pp. 458-462, Feb. 2007. 